\title{
ORIENTACIÓN DE LAS FLORES DE DOS POBLACIONES NORTEÑAS DE PACHYCEREUS PECTEN-ABORIGINUM (CACTACEAE)
}

\author{
Ileem Aguilar-Gastelum ${ }^{1}$ y Francisco Molina-Freaner ${ }^{2,3}$ \\ 'Licenciatura en Biología, Universidad de Sonora, Hermosillo, Sonora, México \\ ${ }^{2}$ Departamento de Ecología de la Biodiversidad, Instituto de Ecología, Universidad Nacional Autónoma de México, \\ Hermosillo, Sonora, México \\ ${ }^{3}$ Autor para la correspondencia: freaner@unam.mx
}

\begin{abstract}
Resumen: Varios estudios previos han documentado que algunas cactáceas columnares muestran orientación ecuatorial de sus flores. Sin embargo, se desconoce qué tan general es este patrón de orientación entre las cactáceas que producen flores laterales. Pachycereus pecten-aboriginum es una especie que produce flores laterales y que tiene una amplia distribución en México. En este trabajo se describe el patrón de distribución de las flores de dos poblaciones norteñas de P. pecten-aboriginum y se explora su relación con la intercepción de radiación fotosintéticamente activa (RFA). Se eligieron dos poblaciones de P. pecten-aboriginum del estado de Sonora, donde se midió el azimut de las costillas y el número de estructuras reproductivas por costilla. Para una de las poblaciones se estimó la RFA interceptada por las costillas de diferente orientación, se colectaron flores orientadas al este, sur y oeste, y se contó el número de óvulos por flor. Las flores de ambas poblaciones tuvieron un promedio de orientación de $191.5^{\circ}$ $\pm 69.7^{\circ}$ y $194.6^{\circ}+61.8^{\circ}$, mostrando una orientación significativa hacia el sur. El análisis del número de óvulos por flor no mostró diferencias significativas entre costillas de diferente orientación. Los valores diarios de intercepción de RFA mostraron que las costillas orientadas hacia el sur y el ápice, son las superficies que más radiación interceptan, mientras que las costillas orientadas hacia el norte son las que menos interceptan. Los resultados se comparan con los datos de otras especies y se discute el posible mecanismo involucrado en la orientación de las flores de esta especie.
\end{abstract}

Palabras clave: cactos columnares, orientación de las flores, Pachycereus, radiación fotosintéticamente activa, Sonora.

\begin{abstract}
Previous studies have documented that flowers of some columnar cacti show equatorial orientation. However, it is unknown how general is this pattern among columnar cacti that produce lateral flowers. Pachycereus pecten-aboriginum is a species that produce lateral flowers and has a wide distribution in Mexico. In this paper we describe the pattern of distribution of flowers across ribs from two northern populations of P. pecten-aboriginum, and explore its relationship with photosynthetic active radiation (PAR) interception. We measured the azimuth and number of reproductive structures of ribs from stems of several plants per population. For one of the populations, we measured PAR for ribs of different orientation and counted the number of ovules per ovary in flowers in eastern, southern, and western positions. Flowers from both populations had a mean orientation of $191.5^{\circ}$ $\pm 69.7^{\circ}$ and $194.6^{\circ}+61.8^{\circ}$, showing a significant southern orientation. The analysis of the number of ovules per ovary indicated no significant differences among ribs. Daily values of PAR interception showed that southern ribs and the apex received greater values, while northern ribs received the lowest values. Our results are compared with other cacti and we discuss the possible mechanism underlying flower orientation in this species.
\end{abstract}

Key words: columnar cacti, flower orientation, Pachycereus, photosynthetic active radiation, Sonora.

$\mathbf{L}$ as angiospermas muestran una gran diversidad en el tamaño, forma, longevidad, color y disposición espacial de sus flores (Willmer, 2011). El tamaño varía desde menos de $1 \mathrm{~mm}$ para las flores de Wolffia, hasta un poco más de un metro para las flores de Rafflesia (Bowman, 1997); mientras que la longevidad varía desde unas cuantas horas hasta cerca de un mes (Primack, 1985). Estas estructuras reproductivas pueden producirse de manera solitaria o agrupadas en inflorescencias, pueden tener una orientación fija o tener movimientos diurnos o estacionales (Zhang et al., 2010; Willmer, 2011). Se ha postulado que la diversidad de los atributos florales está relacionada con diversos factores bióticos (polinizadores) y abióticos (temperatura, radiación solar) del ambiente donde se desarrollan (Willmer, 2011). 
La orientación de las flores puede estar influenciada por factores bióticos. Por ejemplo, las flores de Commelina communis se disponen horizontalmente de manera natural (Ushimaru et al., 2009), mientras que las de Erythronium japonicum se orientan hacia la parte inferior de las laderas (Ushimaru et al., 2006). Al modificar experimentalmente la orientación natural de las flores de ambas especies se redujo el reconocimiento por parte de los polinizadores y disminuyó la tasa de visita. Las flores con ángulo modificado tuvieron menor transferencia de polen y experimentaron una reducción de la fecundidad. Estos resultados fueron interpretados como evidencia de que los polinizadores ejercen una presión selectiva sobre la orientación de las flores (Ushimaru et al., 2006, 2009).

De igual forma, diversos estudios han documentado que varios factores abióticos afectan la orientación de las flores. Por ejemplo, las flores de Anisodus luridus son colgantes; es decir, se orientan hacia el suelo (Wang et al., 2010). Al modificar experimentalmente la orientación natural de estas flores, Wang et al. (2010) detectaron que la lluvia lava el estigma, reduce la captura de polen y reduce la germinación del polen en el estigma debido a la exposición directa a la radiación solar. Esta evidencia sugiere que en ambientes donde las lluvias son frecuentes o con niveles elevados de radiación solar, las flores colgantes pueden tener ventajas sobre las flores erectas (Wang et al., 2010).

En el caso de algunas cactáceas columnares, se ha documentado que las flores muestran una orientación ecuatorial y se ha postulado que esta orientación ecuatorial está estrechamente relacionada con la intercepción de radiación fotosintéticamente activa (RFA; Tinoco-Ojanguren y MolinaFreaner, 2000). Los tallos de la mayoría de las especies de cactáceas columnares son de crecimiento vertical y la incidencia de RFA en este tipo de superficies no se distribuye de manera uniforme entre las diferentes costillas. En este tipo de tallos verticales, la radiación que incide en las costillas es menor comparativamente que la que incide en superficies horizontales. La radiación que interceptan las diferentes costillas de este tipo de tallos varía con la latitud y la estación del año (Nobel, 1988). Por ejemplo, para los $30^{\circ}$ de latitud norte durante el equinoccio, las costillas orientadas hacia el sur reciben más de 30 moles $\mathrm{m}^{-2}$ día $^{-1}$, aquellas orientadas hacia el este o el oeste reciben entre 20 y 30 moles $^{-2}$ día $^{-1}$, mientras que las orientadas hacia el norte reciben menos de 10 moles $\mathrm{m}^{-2}$ día $^{-1}$ (Nobel, 1988). En general, en las regiones subtropicales del hemisferio norte y sur, las costillas con orientación ecuatorial interceptan mayor RFA que las costillas con orientación polar (Nobel, 1988). Esta asimetría en la intercepción de RFA está asociada con la distribución de las flores de algunas especies (Tinoco-Ojanguren y MolinaFreaner, 2000).

Las flores de las cactáceas columnares pueden producirse en el ápice del tallo como en el caso de Carnegiea gigantea (Johnson, 1924), a lo largo de las costillas en una posición lateral como en Pachycereus pringlei (TinocoOjanguren y Molina-Freaner, 2000), o en estructuras especializadas como el cefalio de Cephalocereus columna-trajani (Valverde et al., 2007). Nuestro conocimiento sobre la orientación de las flores de las cactáceas columnares se restringe a unas cuantas especies. Para el caso de las cactáceas columnares sudamericanas, Trichocereus chilensis y Eulynchia castaneae, Rundel (1974) mostró que las flores están orientadas hacia el norte; es decir, muestran orientación ecuatorial. Para el caso de las cactáceas columnares norteamericanas, varios estudios han documentado que las flores se orientan hacia el sur; esto es, también tienen una orientación ecuatorial. Johnson (1924) estudió las flores apicales de Carnegiea gigantea y detectó que estas se producen principalmente hacia el sureste. Para el caso de Pachycereus pringlei, Tinoco-Ojanguren y Molina-Freaner (2000), mostraron que las flores se producen principalmente en las costillas orientadas hacia el sur, con un promedio de orientación de $190^{\circ}$.

Una de las hipótesis que se ha planteado para explicar la orientación ecuatorial, propone que la mayor intercepción de RFA en las caras ecuatoriales de los tallos provoca una mayor ganancia de $\mathrm{CO}_{2}$ y una temperatura más propicia para la inducción de las areolas a producir flores (TinocoOjanguren y Molina-Freaner, 2000). Los estudios, en torno a la fotosíntesis de cactáceas, indican que el punto de compensación de luz se alcanza a los $5 \mathrm{~mol} \mathrm{~m}^{-2}$ día $^{-1}$, mientras que el punto de saturación se alcanza a los $20-30 \mathrm{~mol} / \mathrm{m}^{-2}$ día $^{-1}$ (Nobel, 1988). Los estudios sobre los patrones de intercepción de RFA en los tallos de Pachycereus pringlei indican que las costillas orientadas hacia el sur reciben valores cercanos al punto de saturación, mientras que las costillas orientadas hacia el norte reciben valores cercanos al punto de compensación (Tinoco-Ojanguren y Molina-Freaner, 2000). Bajo este escenario, las costillas orientadas al sur interceptan mayor RFA, alcanzan tasas más altas de ganancia de carbono y producen más flores. En contraste, en las costillas orientadas hacia el norte, la ganancia de carbono es mínima y por ende, producen muy pocas flores (TinocoOjanguren y Molina-Freaner, 2000).

Estos estudios previos parecerían indicar que las cactáceas columnares muestran orientación ecuatorial de sus flores. Sin embargo, no se conoce que tan general es este patrón de orientación entre las cactáceas que producen flores laterales a lo largo de las costillas. Pachycereus pectenaboriginum es una especie que produce flores laterales y que tiene una amplia distribución en México (Bravo-Hollis, 1978). Además, se desconoce si esta especie muestra un patrón de orientación de sus flores similar al reportado para P. pringlei (Tinoco-Ojanguren y Molina-Freaner, 2000). En este trabajo se describe el patrón de distribución de las flores de P. pecten-aboriginum y se explora su relación con la intercepción de RFA de los tallos. 
Orientación de las flores de una CACtácea columnar del norte de MéXico.

\section{Material y métodos}

Especie de estudio. Pachycereus pecten-aboriginum es una cactácea columnar endémica de México, se distribuye desde Oaxaca hasta Sonora, a lo largo de la costa del Pacífico (Bravo-Hollis, 1978). En el estado de Sonora se distribuye en las selvas secas y los matorrales espinosos del sur y centro de la entidad (Turner et al., 1995). En esta región, la especie florece de diciembre a marzo y fructifica en junio y julio (Turner et al., 1995). Las flores abren en la noche y son visitadas por murciélagos nectarívoros (Valiente-Banuet et al., 2004). En el estado de Sonora, las flores permanecen abiertas durante el día siguiente y son visitadas por aves y abejas (Molina-Freaner et al., 2004b).

Sitios de estudio. Se eligieron dos poblaciones representativas del extremo norte de la distribución de la especie, para el presente estudio: rancho El Diamante y Masiaca (Figura 1). El rancho El Diamante se localiza al sureste de Hermosillo, Sonora, en el km 84 de la carretera Hermosillo-Yécora ( $28^{\circ} 41.74^{\prime} \mathrm{N}$; $\left.110^{\circ} 15.83^{\prime} \mathrm{O}\right)$, con una altitud de $370 \mathrm{~m}$ s.n.m. La vegetación corresponde a matorral espinoso y la precipitación media anual es de $431 \mathrm{~mm}$ (Molina-Freaner et al., 2004a; Morales-Romero y Molina-Freaner, 2008). La otra población se encuentra ubicada cerca del pueblo de Masiaca $\left(26^{\circ} 44.72^{\prime} \mathrm{N} ; 109^{\circ} 12.05^{\prime} \mathrm{O}\right)$, al sur del estado, a una altitud de $93 \mathrm{~m}$ s.n.m. La vegetación corresponde a matorral espinoso y la precipitación media anual es de 370 mm (Servicio Meteorológico Nacional, sección normales climatológicas, Sonora y Masiaca, respectivamente. http:// smn.cna.gob.mx/index.php?option=com_content $\&$ view $=$ ar ticle $\&$ id $=42 \&$ Itemid $=75$ )

Tamaño de las plantas y número de estructuras reproductivas. Se eligieron 17 plantas adultas en floración en Masiaca

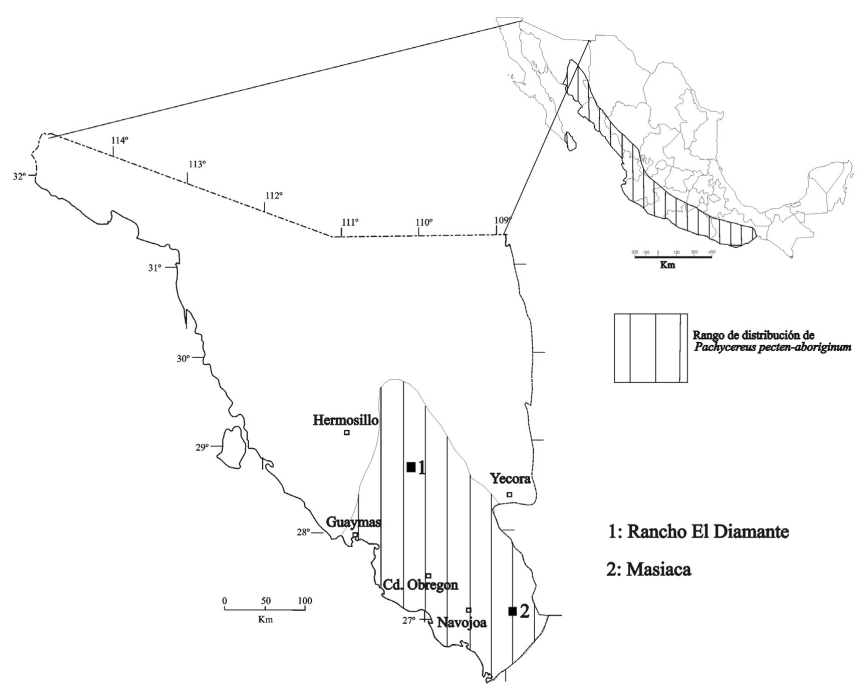

Figura 1. Localización de las dos poblaciones de Pachycereus pecten-aboriginum estudiadas en este trabajo. y 14 plantas adultas en floración en el Rancho El Diamante, para evaluar la distribución de las flores. Con el objeto de caracterizar el tamaño de las plantas estudiadas de las dos poblaciones, se midió la altura como la longitud del eje principal o la altura de la ramificación más alta, y el número de ramificaciones por planta. La altura se midió con una varilla de medición telescópica con un dispositivo que descansa en el ápice del tallo. La longitud de las ramas con estructuras reproductivas fue medida como la distancia entre el ápice y el punto de inserción de la rama en el eje principal. En cada rama reproductiva se contó el número de costillas a lo largo del tallo; así como el azimut de las costillas, usando una brújula Brunton 5008 (Riverton, WY, USA). Para cada costilla, se contó el número de botones florales, flores o frutos en desarrollo durante la fase de floración.

Debido a que existe evidencia de que en otra especie de Pachycereus, las flores y frutos con orientación ecuatorial producen más óvulos y semillas (Figueroa-Castro y Valverde, 2011), se tomó una muestra de flores con diferente orientación de la población del rancho El Diamante, para evaluar si $P$. pecten-aboriginum muestra un patrón similar. La muestra consistió de 25 flores de diferente orientación: entre $70^{\circ}$ y $132^{\circ}$, entre $133^{\circ}$ y $195^{\circ}$ y, entre $196^{\circ}$ y $258^{\circ}$. Estos rangos se eligieron con base en la disponibilidad de flores durante el muestreo. Durante este muestreo no fue posible ubicar flores orientadas hacia el norte. Estas flores fueron llevadas al laboratorio donde se midió el diámetro longitudinal del ovario y se hizo un conteo del número de óvulos por ovario, para evaluar si existen diferencias significativas en su potencial reproductivo.

Caracterización de la radiación fotosintéticamente activa interceptada por las costillas con diferente orientación. Se eligió una planta de la población del rancho El Diamante para medir la radiación fotosintéticamente activa (RFA) que interceptan las costillas con diferente orientación durante la fase de floración. La planta tenía un sólo tallo (sin ramas), medía $2.60 \mathrm{~m}$ de altura y $10.3 \mathrm{~cm}$ de diámetro basal, tenía botones y flores y estaba alejada de otros árboles que pudieran provocar sombra a su tallo. Las mediciones se realizaron del 22 al 26 de diciembre de 2010, intervalo que presentó días soleados y nublados. La RFA se midió usando sensores de luz conocidos como fotodiodos, que se calibraron usando un sensor cuántico de luz LICOR 190SB (LI-COR, Lincoln, NE, USA) (Tinoco-Ojanguren y Molina-Freaner, 2000). Los sensores se colocaron en la cresta de diferentes costillas y $50 \mathrm{~cm}$ abajo del ápice del tallo, en la zona donde se producen las flores. Las costillas elegidas correspondían aproximadamente a las orientaciones norte, oeste, sur y este. La RFA que intercepta el ápice se midió con un sensor cuántico de luz (LICOR 190SB) colocado en al ápice de la planta. Los datos se tomaron cada 5 segundos y se obtuvo un promedio cada 5 minutos, los cuales se almacenaron en un registrador (Campbell 21X, Campbell Scientific, Logan, 
UT, USA) de datos tipo micrologger (Tinoco-Ojanguren y Molina-Freaner, 2000).

Análisis de datos. Para el análisis del tamaño de las plantas, las ramas y el número de costillas, se calculó el promedio, la desviación estándar y el rango de variación de cada población. Los datos de RFA de todos los días registrados en la planta del rancho El Diamante se integraron para calcular el promedio y la desviación estándar de intercepción diaria asociado a cada orientación de costilla. Para el análisis de la orientación de las estructuras reproductivas, se usó el paquete estadístico ORIANA (Kovach, 2013) para calcular el promedio y la desviación estándar del azimut de las flores. Para evaluar la orientación de las flores, se usó la prueba de Rayleigh, la cual evalúa si las flores tienen una distribución uniforme o si muestran una orientación significativa (Zar, 1999).

\section{Resultados}

Las 14 plantas adultas muestreadas en la población del rancho El Diamante variaron en altura de 4.8 a $8.4 \mathrm{~m}$, con una media ( \pm 1 d.e.) de $6.4 \pm 1.2 \mathrm{~m}$; y tuvieron de 9 a 62 ramificaciones, con un promedio de $28.8 \pm 17.8$ ramas por planta. Las ramas tuvieron en promedio una longitud de $5.2 \pm 0.9$ m y $10.8 \pm 0.8$ costillas. En promedio, las plantas de esta población produjeron $8.2 \pm 6.5$ estructuras reproductivas. Las 17 plantas adultas muestreadas en Masiaca variaron en altura de 4.5 a $6.5 \mathrm{~m}$, con un promedio de $5.3 \pm 0.6 \mathrm{~m}$; y tuvieron de 7 a 61 ramificaciones, con una media de $26.0 \pm$ 14.3. Las ramas tuvieron en promedio una longitud de $4.5 \pm$ $0.7 \mathrm{~m}$ y $10.5 \pm 0.7$ costillas. En esta población, las plantas produjeron $6.9 \pm 6.3$ estructuras reproductivas.

En la población del rancho El Diamante, las estructuras reproductivas mostraron una mayor frecuencia entre los $90^{\circ}$ y $270^{\circ}$ de azimut (Figura 2a). El análisis de estadística descriptiva de los datos circulares arrojó un promedio de $191.5^{\circ}$ y una desviación estándar de $69.7^{\circ}$, lo cual sugiere que las flores se orientan principalmente al sur. La prueba de Rayleigh dio un valor de $170.5(P<0.0001, \mathrm{n}=750)$, lo que indica que para esta población las flores tienen una orientación significativa hacia el sur.

En la población de Masiaca, las estructuras reproductivas mostraron un patrón similar. La mayor parte de las flores se producen entre $\operatorname{los} 90^{\circ}$ y $270^{\circ}$ (Figura 2b). En esta población las flores tuvieron una orientación promedio de $194.6^{\circ}$ $\pm 61.8^{\circ}$. La prueba de Rayleigh tuvo un valor de $59.3(P$ $<0.0001, \mathrm{n}=191$ ), indicando que para esta población las flores muestran una orientación significativa hacia el sur.

Las flores provenientes de costillas orientadas hacia el este, sur y oeste en la población del rancho El Diamante no mostraron diferencias en su potencial reproductivo (Figura 3). El número de óvulos por ovario fluctúo de 429 a 909 para la muestra de flores de esta población. El análisis
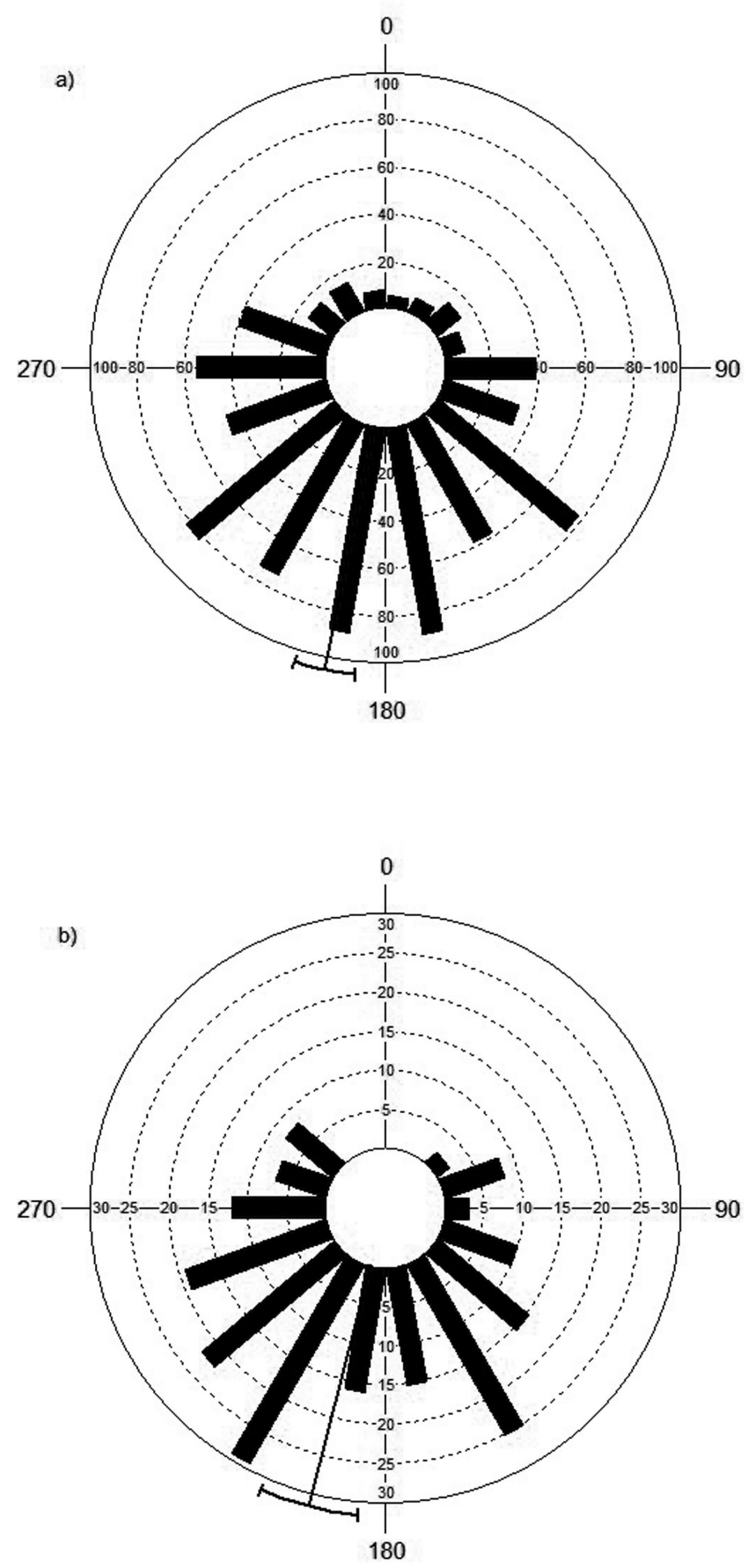

Figura 2. Histograma circular que muestra la distribución de las estructuras reproductivas en los tallos de P. pecten-aboriginum de la población del rancho El Diamante (a) y en Masiaca (b), en el estado de Sonora.

estadístico ( $\mathrm{F}=1.65 ; \mathrm{P}>0.05)$ mostró que no hubo diferencias significativas entre los grupos de flores de diferente orientación (Figura 3). En cambio, el análisis de la relación entre el tamaño del ovario y el número de óvulos mostró una relación significativa entre el diámetro longitudinal y el número de óvulos $(\mathrm{F}=5.65 ; P<0.05)$. 


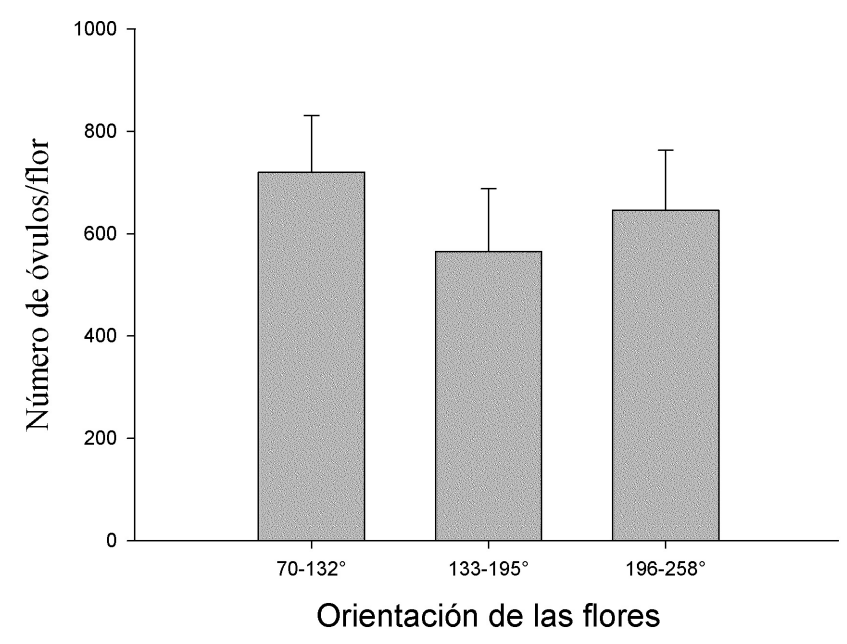

Figura 3. Promedio ( \pm d.e.) del número de óvulos por ovario en las flores de diferente orientación en los tallos de la población de $P$. pecten-aboriginum del rancho El Diamante.

La integración de los valores diarios de RFA interceptada por las costillas durante cinco días muestra que las costillas orientadas al sur y el ápice son las superficies que mayor RFA reciben (Figura 4). Estas dos superficies reciben en promedio aproximadamente 27 moles $\mathrm{m}^{-2} \mathrm{dí}^{-1}$. En cambio, las costillas orientadas al este y oeste reciben en promedio entre 8 y 10 moles $\mathrm{m}^{-2} \mathrm{dí}^{-1}$, mientras que la costilla orientada al norte recibe sólo 5 moles $\mathrm{m}^{-2}$ día ${ }^{-1}$.

\section{Discusión}

Los resultados de este estudio indican que en las dos poblaciones estudiadas de Pachycereus pecten-aboriginum, las estructuras reproductivas se producen principalmente en las costillas orientadas hacia el sur. Este patrón de orientación coincide con la superficie del tallo (costilla) que intercepta más RFA en la planta estudiada en el rancho El Diamante. Esta orientación ecuatorial es similar a la detectada para $P$. pringlei en la región del desierto Sonorense, y congruente con los resultados obtenidos para otras especies de cactáceas columnares, tanto en el hemisferio norte como en el hemisferio sur (Rundel, 1974; Tinoco-Ojanguren y MolinaFreaner, 2000).

Los resultados de intercepción de RFA indican que las costillas orientadas al sur y el ápice del tallo reciben niveles de RFA cercanos al punto de saturación, mientras que las costillas orientadas al norte reciben niveles cercanos al punto de compensación. Si la inducción de las areolas a producir botones florales depende de la acumulación de carbohidratos y no existe translocación entre costillas, se espera que la superficie fotosintética que intercepte más luz sea capaz de fijar más carbono y los recursos necesarios para inducir la producción de flores. Los estudios sobre la fijación de $\mathrm{CO}_{2}$ en la superficie fotosintética de tallos de cactos columnares muestra que el punto de saturación se alcanza a 30 moles $\mathrm{m}^{-2} \mathrm{dia}^{-1}$, mientras que el punto de compensación se alcanza a los 3-4 moles $\mathrm{m}^{-2}$ dia $^{-1}$ (Nobel, 1988). Para el caso de $\mathrm{Pa}$ chycereus pecten-aboriginum, no se ha documentado la respuesta fotosintética a la luz en las poblaciones naturales. Sin embargo, si la respuesta es similar a la de otras cactáceas columnares que han sido estudiadas (Nobel, 1988; Bronson et al., 2011), se espera que las costillas orientadas hacia el sur experimenten mayor ganancia de carbono y acumulen suficientes reservas para inducir a las areolas a producir flores. Es probable que este sea el mecanismo responsable de la orientación ecuatorial de las flores de algunas especies de cactáceas columnares. Sin embargo, es necesario estudiar con detalle la respuesta fotosintética a la RFA para establecer la relación existente entre la intercepción de luz, la captura de carbono y la producción de flores de las costillas de diferente orientación, para evaluar esta hipótesis.

Nuestros resultados sobre intercepción de RFA tienen varias limitaciones. La planta donde se midió la RFA en el rancho El Diamante era de menor altura que las plantas donde se documentó la orientación de las flores. Por otra parte, la planta tenía un sólo tallo y no tenía ramas ni vegetación cercana que provocara sombra en el tallo. La intercepción de RFA por los tallos de cactáceas puede disminuir por el sombreado de las espinas, las ramificaciones de la planta, la vegetación circundante y por la topografía (Nobel, 1988). La intercepción de RFA en la planta estudiada representa la incidencia de luz para un tallo aislado de la población. Por esta razón, el estudio de la intercepción de RFA en los tallos de una especie como Pachycereus pecten-aboriginum, la cual puede llegar a tener más de 60 ramas, requiere de un mayor número de sensores en varias plantas que documenten la variabilidad provocada por las ramas de la misma planta y la vegetación circundante.

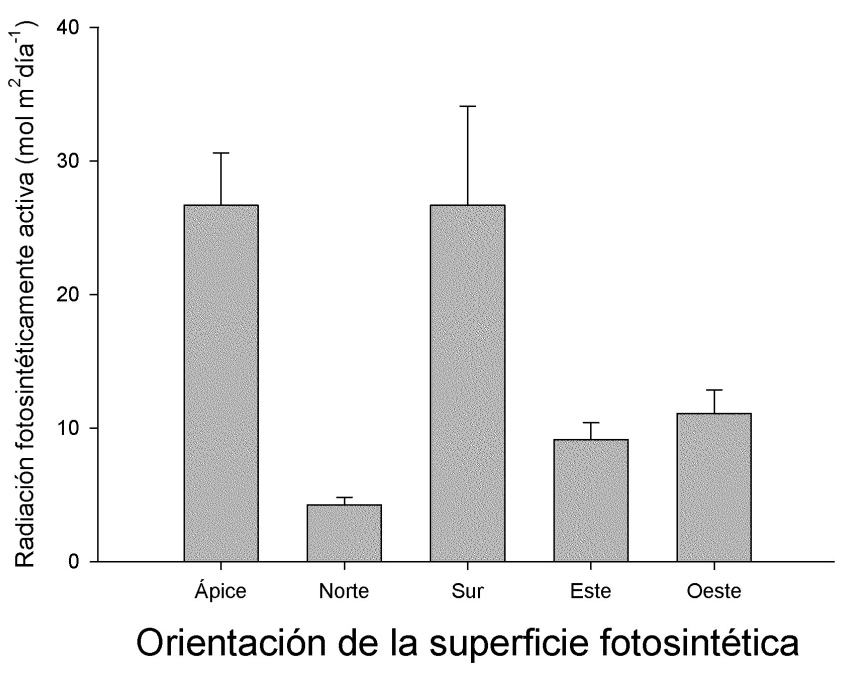

Figura 4. Promedio (+ d.e.) de la intercepción diaria de radiación fotosintéticamente activa (RFA) de costillas de diferente orientación durante el periodo de estudio en una planta del rancho El Diamante. 
Se ha postulado que la orientación de las flores de los cactos columnares también tiene una influencia en el éxito reproductivo (Figueroa-Castro y Valverde 2011). Según estos autores, si la disponibilidad de recursos para las flores difiere entre costillas, se esperaría encontrar diferencias en el número de óvulos por flor, así como en el número y peso de las semillas producidas por fruto de diferente orientación. Para el caso de Pachycereus weberi, estos autores encontraron evidencia de que las flores orientadas hacia el sur producen más óvulos y los frutos provenientes de estas flores producen más semillas y de mayor peso. En contraste, nuestros resultados sobre el número de óvulos por ovario de flores de P. pecten-aboriginum provenientes de las costillas del este, sur y oeste no apoyan la hipótesis de que las flores de orientación ecuatorial tienen mayor potencial reproductivo. El muestreo no incluyó flores de las costillas del norte y por tanto, los resultados deben verse con reserva hasta que la evaluación incluya flores de todas las orientaciones. Sin embargo, los datos parecen indicar que el número de óvulos por ovario depende más del tamaño del ovario que la orientación de las flores. En el caso de este estudio, no se evaluó el número de semillas por fruto asociado a diferente orientación, debido a que durante el 2011, una helada dañó a los frutos en desarrollo durante el mes de febrero, y al final de la estación reproductiva, ningún fruto llegó a madurar. Por esta razón, no se pudo evaluar si los frutos formados en las costillas orientadas al sur producen más semillas y de mayor peso.

Los resultados indican que las flores de las poblaciones norteñas de Pachycereus pecten-aboriginum tienen una orientación ecuatorial. Esta especie tiene una amplia distribución en México, desde Oaxaca hasta Sonora (Bravo-Hollis, 1978). A lo largo de su rango de distribución, el patrón de intercepción de RFA de sus tallos puede modificarse debido a la influencia de la latitud, así como a la altura y cobertura de la vegetación (Nobel, 1988). Por esta razón, es necesario documentar la orientación de sus flores en el sur de su distribución, para evaluar si existe variación geográfica en el patrón de orientación. Finalmente, para acrecentar el conocimiento sobre los factores responsables de la orientación de las flores de cactáceas columnares, es necesario estudiar con mayor detalle la respuesta fisiológica de las costillas a la RFA y el mecanismo que induce a las areolas a producir estructuras reproductivas.

\section{Agradecimientos}

Este trabajo constituye la tesis que para obtener el grado de Biólogo presentó Ileem Aguilar en la Universidad de Sonora. Agradecemos a dos revisores por sus comentarios y críticas constructivas, a José F. Martínez y Alina Santos por la ayuda en el campo y laboratorio, así como a Clara Tinoco por sus críticas al texto y su asesoría en las mediciones de luz.

\section{Literatura citada}

Bowman J.L. 1997. Evolutionary conservation of angiosperm flower development at the molecular and genetic level. Journal of Biosciences 22:515-527.

Bravo-Hollis H. 1978. Las Cactáceas de México. Vol. 1. $2^{\mathrm{a}}$ ed. Universidad Nacional Autónoma de México, México, D.F.

Bronson D.R., English N.B., Dettman D.L. y Williams D.G. 2011. Seasonal photosynthetic gas exchange and water-use efficiency in a constitutive CAM plant, the giant saguaro cactus (Carnegiea gigantea). Oecologia 167:861-871.

Figueroa-Castro D.M. y Valverde P.L. 2011. Flower orientation in Pachycereus weberi (Cactaceae): effects on ovule production, seed production and seed weight. Journal of Arid Environments 75:1214-1217.

Johnson D.S. 1924. The influence of insolation on the distribution and on the developmental sequence of the flowers of the giant cactus of Arizona. Ecology 5:70-82.

Kovach W.L. 2013. Oriana version 4. Kovach Computing Services, Anglesey.

Molina-Freaner F., Castillo G.R., Tinoco-Ojanguren C. y Castellanos A.E.V. 2004a. Vine species diversity across environmental gradients in northwestern Mexico. Biodiversity and Conservation 13:1853-1874.

Molina-Freaner F., Rojas-Martínez A., Fleming T.H. y ValienteBanuet A. 2004b. Pollination biology of the columnar cactus Pachycereus pecten-aboriginum in north-western México. Journal of Arid Environments 56:117-127.

Morales-Romero D. y Molina-Freaner F. 2008. Influence of buffelgrass pasture conversion on the regeneration and reproduction of the columnar cactus, Pachycereus pecten-aboriginum in northwestern Mexico. Journal of Arid Environments 72:228-237.

Nobel P.S. 1988. Environmental biology of agaves and cacti. Cambridge University Press, Cambridge.

Primack R.B. 1985. Longevity of individual flowers. Annual Review of Ecology and Systematics 16:15-37.

Rundel P.W. 1974. Trichocereus in the mediterranean zone of central Chile. Cactus and Succulent Journal (US) 46:86-88.

Tinoco-Ojanguren C. y Molina-Freaner F. 2000. Flower orientation in Pachycereus pringlei. Canadian Journal of Botany 78:1489-1494.

Turner R.M., Bowers J.E y Burgues T.L. 1995. Sonoran Desert Plants: An Ecological Atlas. University of Arizona Press, Tucson.

Ushimaru A., Kawase D. y Imamura A. 2006. Flowers adaptively face down-slope in 10 forest-floor herbs. Functional Ecology 20:585-591.

Ushimaru A., Dohzono I., Takami Y. y Hyodo F. 2009. Flower orientation enhances pollen transfer in bilaterally symmetrical flowers. Oecologia 160:667-674.

Valiente-Banuet A., Molina-Freaner F., Torres A., Arizmendi M.C. y Casas A. 2004. Geographic differentiation in the pollination system of the columnar cactus Pachycereus pectin-aboriginum. American Journal of Botany 91:850-855.

Valverde P.L., Vite F., Pérez-Hernández M.A. y Zavala-Hurtado J.A. 2007. Stem tilting, pseudocephalium orientation, and stem allometry in Cephalocereus columna-trajani along a short latitudinal gradient. Plant Ecology 188:17-27.

Wang Y., Meng L.L., Yang Y.P., y Duan Y.W. 2010. Change in floral orientation in Anisodus luridus (Solanaceae) protects pollen 
Orientación de las flores de una Cactácea Columnar del norte de México.

grains and facilitates development of fertilized ovules. American Journal of Botany 97:1618-1624.

Willmer P. 2011. Pollination and Floral Ecology. Princeton University Press, Princeton.

Zar J.H. 1999. Biostatistical Analysis. 4ª ed. Prentice Hall, Upper
Saddle River.

Zhang S., Ai H.L., Yu W.B., Wang H., y Li D.Z. 2010. Flower heliotropism of Anemone rivularis (Ranunculaceae) in the Himalayas: effects of floral temperature and reproductive fitness. Plant Ecology 209:301-312.

Recibido: 21 de noviembre de 2013

Aceptado: 5 de febrero de 2014 